\title{
Aconselhamento Genético: Análise e Contribuições a partir do Modelo de Aconselhamento Psicológico
}

Genetic Counseling: Analysis and Contributions from the Model of Psychological Counseling

Asesoramiento Genético: Análisis y Contribuciones a partir del Modelo de Asesoramiento Psicológico

Juliana Santos Souza Hannum, Fábio Jesus Miranda, Luciana Novais de Oliveira Brito, Sebastião Benício da Costa Neto \& Aparecido Divino da Cruz

Pontifícia Universidade Católica de Goiás

http://dx.doi.org/10.1590/1982-3703001372013 
Resumo: O Aconselhamento Genético (AG) constitui um processo de investigação do diagnóstico de doenças genéticas. Este estudo de caso objetivou a análise de um modelo de AG. A coleta de dados foi realizada por meio de entrevista semiestruturada e da observação de uma sessão de AG. Os participantes foram profissionais biomédicos e a consulente de uma criança com diagnóstico de síndrome do duplo Y. Verificou-se que o AG envolve uma relação intersubjetiva complexa. Percebeu-se, nos dois polos da relação - profissional e consulentefamiliar -, os aspectos emocionais (angústia, temor, culpas etc.) e defensivos (identificação, racionalização etc.) referidos na literatura das crises vitais. A análise do processo de AG indicou: 1) dissonância entre a teoria do AG e a ação do profissional; 2) fixação (defensiva) ao protocolo do AG; 3) uso de linguagem técnica dificultando contato e acolhimento do consulente; e, 4) tempo de compreender considerado na dimensão cronológica e não lógico. Infere-se que os fatores afetivos (angústia, temor, culpas etc.) e a defesa psíquica (identificação, racionalização) restringiram a comunicação do diagnóstico comprometendo o acolhimento e esclarecimento do contexto vital do consulente. Conclui-se que o modelo de AG poderia se enriquecer com a experiência do modelo de aconselhamento psicológico no que se refere ao manejo de crises vitais e no trabalho em equipes multidisciplinares.

Palavras-chave: Aconselhamento Genético. Família. Medicina do Comportamento.

Abstract: Genetic Counseling (GC) comprises an investigation regarding the diagnosis of genetic diseases. Thus, this case study aimed to analyze a particular GC model. Data collection was conducted through semi-structured interviews and an observation of a GC session. The participants comprised professionals, a biomedical team, and a consultant for a child diagnosed with double $Y$ syndrome. It was observed that GC involves a complex intersubjective relationship and that, in both ends of the relationship (i.e., professional consultants and family members), emotional factors (anxiety, fear, guilty, etc.) and defensiveness (identification, rationalization, etc.) restricted the communication of the diagnosis, preventing the host from effectively explaining such vital information to the inquirer. The GC review process also indicated the following four findings: 1) A dissonance between GC theory and professional actions, 2) A fixation on GC protocol, 3) The use of technical language that made it difficult to communicate, and 4) Insufficient time for considering the chronological dimension. On the basis of the findings, it can be concluded that the GC model could be enriched by the psychological counseling model, especially in relation to crisis management and the vital work of multidisciplinary teams.

Keywords: Genetic Counseling. Family. Behavior Medicine.

Resumen: El Asesoramiento Genético (AG) constituye un proceso de investigación del diagnóstico de enfermedades genéticas. Este estudio de caso tuvo como objetivo analizar un modelo de AG. La colecta de datos fue realizada por medio de entrevista semiestructurada y de la observación de una sesión de AG. Los participantes fueron profesionales biomédicos y el consultante de un niño con diagnóstico de síndrome del doble Y. Se verificó que el AG implica una relación intersubjetiva compleja. Se percibió, en los dos polos de la relación - profesional y consultante - familiar -, los aspectos emocionales (angustia, temor, culpas, etc.) y defensivos (identificación, racionalización etc.) mencionados en la literatura como crisis vitales. El análisis del proceso de AG indicó: 1) disonancia entre la teoría del AG y la acción del profesional; 2) fijación (defensiva) al protocolo del AG, 3) uso de lenguaje técnico dificultando contacto y acogimiento del consultante; e, 4) tiempo de comprender considerado en la dimensión cronológica y no lógica. Se infiere que los factores afectivos (angustia, temor, culpas, etc) y la defensa psíquica (identificación, racionalización) restringieron la comunicación del diagnóstico comprometiendo el acogimiento y aclaratoria del contexto vital del consultante. Se concluye que el modelo de AG podría enriquecerse con la experiencia del modelo de asesoramiento psicológico en lo que se refiere al manejo de crisis vitales y en el trabajo en equipos multidisciplinarios.

Palabras clave: Asesoramiento Genético. Familia. Medicina de la Conducta. 


\section{Introdução}

Serviços de Aconselhamento Genético (AG) têm, entre seus vários objetivos, a definição do diagnóstico, a informação à família sobre o diagnóstico e seu prognóstico e sobre o risco de ocorrência e/ou recorrência do desfecho sobre o qual se aconselha. Devem também orientar os pais quanto às características do desenvolvimento do filho e quais os atendimentos e tratamentos que se fazem necessários para o melhor desenvolvimento da criança. O AG é indicado para toda família afetada com distúrbio genético (Rim, Magna, \& Ramalho, 2006).

Durante o processo, o conselheiro genético poderá contribuir para redirecionar as terapias adequadas, tornando-as mais específicas e compatíveis com as peculiaridades da doença e, consequentemente, contribuir de forma eficiente para se eleger as estratégias de intervenção mais pertinentes para cada caso. Há uma estimativa de que $13 \%$ a $41 \%$ dos casos necessitem de suporte psicossocial durante e/ou em decorrência do AG (Bleiker, Hahn, \& Aaronson, 2003).

O AG, como todos os outros procedimentos de atenção à saúde humana, fundamenta-se sob seis princípios éticos básicos (Silva, \& Ramalho, 1997), quais sejam:

I. O princípio da autonomia estabelece que os testes genéticos devam ser estritamente voluntários, resultantes de um aconselhamento apropriado e que proporcione aos consulentes decisões absolutamente pessoais:

II. O princípio da privacidade determina que os resultados dos testes genéticos de um indivíduo não podem ser comunicados a nenhuma outra pessoa sem o seu consentimento expresso aos responsáveis pela pesquisa;

III. O princípio da justiça garante proteção aos direitos de populações vulneráveis, tais como: crianças, pessoas com retardamento mental ou problemas psiquiátricos ou culturais especiais;

IV. O princípio da igualdade rege o acesso igual aos testes independentemente de origem geográfica ou classe socioeconômica;
V. O princípio da qualidade assegura que todos os testes oferecidos devem ter especificidade e sensibilidade adequadas, sendo realizados em laboratórios capacitados e com monitoração profissional e ética; e

VI. O princípio da não maleficência prevê o dever de não causar danos físicos, morais ou psicológicos aos pacientes por meio de intervenções médicas. O princípio da beneficência se liga a este ao preconizar a necessidade da tomada de medidas proativas que levarão ao bem-estar do paciente.

As instituições que oferecem o serviço de AG devem garantir o sigilo e a confidencialidade dos diagnósticos e arquivar a documentação dos seus consulentes e seus prontuários em local privado e seguro. De modo geral, a conduta do conselheiro deve sempre ser pautada pelos princípios gerais da bioética (Garicochea, \& Barros, 2008).

\begin{abstract}
Atualmente, o AG tem merecido destaque como uma prática imprescindível em saúde pública. Esta noção se dá a partir das sessões de AG, nas quais são abordados aspectos que podem variar desde as opções reprodutivas como a discussão sobre riscos e probabilidades de nascimento de crianças com determinados traços genéticos, até cuidados precoces relativos às doenças genéticas de expressão tardia (Brunoni, 2002). Conforme Mc Kusick (1975), as etapas do AG incluem: (1) levantamento da história médica pessoal e familiar, avaliação dos exames clínicos e genéticos já realizados e indicação de outros exames, se necessário; (2) análise dos dados, visando diagnosticar, confirmar ou excluir uma condição genética conhecida; (3) informações acerca da natureza da condição genética identificada e de suas implicações para a saúde física ou mental do indivíduo; (4) esclarecimento sobre o mecanismo de herança e cálculo de risco de ocorrência ou recorrência da condição em irmãos ou filhos de um indivíduo.
\end{abstract}

O AG tem como finalidade ajudar as pessoas a resolverem seus problemas no campo da hereditariedade (Silva, \& Ramalho, 1997). 
Portanto, orienta o consulente e/ou os familiares sobre a tomada de decisões conscientes e equilibradas a respeito da saúde deles. É importante reafirmar que, quanto mais cedo for iniciado o AG, maior será a probabilidade de um melhor prognóstico futuro (Ramalho, Magna, \& Silva, 2002).

O AG, como procedimento de diagnóstico, implica que os conselheiros devem atuar como facilitadores de um processo complexo de entendimento do que está ocorrendo com o consulente e sua família e que ajam para que os consulentes façam um processo de ajuste perante a nova situação a ser vivenciada (Pina-Neto, 2008). Segundo Borges-Osório e Robinson (2001), a informação de ordem genética tem implicações importantes. Além de conter dados médicos e científicos de difícil compreensão, abrange aspectos psicológicos desencadeados pela confirmação do diagnóstico positivo.

As alterações genéticas possuem causas multideterminadas, portanto, não se deve admitir uma intervenção que exclua a dimensão emocional e nem tampouco o contexto socioambiental no qual se insere o consulente e seus familiares. As famílias que atravessam uma problemática de ordem genética precisam do olhar integrador do profissional de saúde mental, de apoio social e de todos os profissionais que estão comprometidos com o seu tratamento. Assim, escutar o consulente e sua família de maneira ampla implica considerar os diferentes fatores que estão potencialmente inter-relacionados e que afetam o problema diagnosticado ou que são afetados por ele. Assim, quando a família se depara com o diagnóstico positivo de uma doença genética e percebe que deverá se adaptar a uma realidade irreversível, envolvendo toda a dinâmica familiar, o casal acaba tendo que revisar antigos desejos, expectativas, planos e sonhos para que novas prioridades sejam estabelecidas (Colmago, \& Biasoli-Alves, 2003).

Estudos têm demonstrado que os familiares somente serão capazes de efetivamente assimilarem as orientações e de oferecerem os atendimentos necessários ao desenvolvimento da criança, quando o choque inicial, provocado pelo diagnóstico positivo para uma doença genética, for elaborado (Evers-Kiebooms, \& Berghe, 1979). A singularidade de cada família influenciará a vivência e a capacidade de chegar ou não à fase de reorganização do pensamento e das atitudes, embora existam fatores reconhecidos como fundamentais na qualidade de elaboração do enfrentamento, tais como o momento do diagnóstico e sua gravidade (Irvin, Kennel, \& Klaus, 1993).

Adicionalmente, o prognóstico, o tipo de cuidado necessário ao consulente, os recursos externos de apoio e as estruturas internas psíquicas disponíveis afetam o modo pelo qual cada indivíduo lida com a situação emergente (Goldberg, Morris, Simmons, Fowler, \& Levison, 1990; Kroeff, Maia, \& Lima, 2000; Pelchat et al., 2001). Tendo em vista as implicações genéticas e sociais dos distúrbios genéticos e o impacto que causam nas famílias, o aparecimento do distúrbio precipita sinais clássicos das crises vitais e é sob esta perspectiva que se deve aborda-lo durante os processos de AG e da conduta clínica de atenção à saúde dos afetados e de seus familiares.

No processo de AG, são vários os temas a serem considerados: a proposição e a análise do teste genético, o contexto sociocultural, o processo de desenvolvimento humano, a relação interpessoal, o processo de luto, o desamparo, os processos de comunicação verbal e não-verbal, os mecanismos de defesa psíquica, os tipos de personalidade, a autoimagem, os modos de enfrentamento, o conceito de saúde e doença etc.

É primordial que os profissionais envolvidos no AG tenham amplo conhecimento de todo o processo por que passam os consulentes, para que possam compreendê-los e lhes dar o tempo necessário para assimilarem as informações e, principalmente, tomarem as decisões necessárias, com clareza e racionalidade (Petean \& Pina-Neto, 1998). Embora se possam visualizar as complexidades envolvidas na tarefa do AG, principalmente no que se refere à dimensão de ordem psicológica, o AG tem se desenvolvido e 
tem sido exercido, principalmente, dentro do campo e da perspectiva das ciências biológicas. Pensa-se que a integração do conhecimento psicológico ao modelo de AG poderia contribuir no atendimento à complexidade de sua função.

O objetivo este trabalho foi analisar um caso de Aconselhamento Genético, com base no modelo do aconselhamento psicológico, buscando caracterizar a dimensão psicológica presente no processo de AG e analisar o modelo atual de AG praticado no Núcleo de Pesquisa Replicon, do Departamento de Biologia da PUC de Goiás.

\section{Método}

\section{Participantes}

Trata-se de estudo de caso descritivo e exploratório. Participaram da pesquisa três profissionais biomédicos geneticistas, de ambos os sexos, de idades entre 37-42, ligados ao NPR; uma consulente de 36 anos, semianalfabeta (apenas sabe escrever o próprio nome), mãe de uma criança de onze anos que apresenta diagnóstico do 47, XYY (Síndrome Caracterizada do Duplo Y) e com retardo mental, dificuldade de aprendizagem e dificuldade na fala.

Como critério de inclusão dos profissionais, foi considerada a experiência maior a um ano de aconselhamento genético e participação voluntária mediante assinatura de um termo de consentimento livre e esclarecido (TCLE). Da família, foi considerado: ter uma criança com o diagnóstico positivo da síndrome 47, XYY e disposição voluntária para assinar o termo de consentimento livre e esclarecido. Foi garantido aos participantes não só o sigilo, como também a possibilidade de desistência da pesquisa a qualquer momento do processo.

\section{Material}

O ambiente do AG foi composto por uma sala de, aproximadamente, $12 \mathrm{~m}^{2}$, com dois sofás, uma mesa e quatro pufes para crianças e preparado para dar privacidade ao processo de AG. O Material utilizado se constituiu de exemplar do roteiro de entrevista semiestruturada, elaborado para a pesquisa, composto por quatro questões que abordavam os seguintes temas: Percepções das Reações Familiares, Percepção da Profissional e Considerações Relevantes para Melhoria do Serviço de AG; Roteiro de entrevista padrão do aconselhamento genético estruturado pela equipe de aconselhamento genético do NPR o qual contém Dados Pessoais e Dados do RN. Os dados do RN requisitado são: Antecedentes Clínicos, Dados Fonoaudiológicos, Consanguinidade, Dados Familiares, Antecedentes Gestacionais, Dados e Medidas do Paciente, Hábitos e Heredograma.

\section{Procedimentos}

Foram realizados contatos com a gerência do NPR e apresentado o projeto de investigação. Após o aceite, os participantes foram recrutados durante a realização dos procedimentos de AG. A eles foram esclarecidos os objetivos da investigação, bem como as condições do TCLE. Os primeiros sujeitos abordados consentiram na participação assinando o TCLE. A coleta de dados foi realizada no segundo semestre de 2010. A pesquisadora inseriu-se na sessão de devolução diagnóstica do AG realizando registro em áudio das interlocuções dos participantes e por meio de observação realizou registros de cada fase do processo. Imediatamente após a sessão de devolução do AG, entrevistouse o conselheiro responsável, registrando as respostas em áudio. A partir da entrevista com o conselheiro e da entrevista de devolução diagnóstica do processo de AG, foi realizada a transcrição literal do material e procedeuse uma síntese temática dos aspectos mais relevantes da fala do conselheiro e da fala do consulente, por meio da técnica de análise de conteúdo de Bardin (2008). Tanto para os profissionais quanto para a família, segundo o que orienta a resolução MS n ${ }^{\circ}$ 196/96 (Brasil, 1996), no que se refere à condução de possíveis adversidades desencadeadas pelo processo de coleta de dados, a equipe se responsabilizou pelo acolhimento de eventuais ocorrências. 


\section{Resultados}

No processo de AG, percebe-se que a consulente possui um vocabulário restrito, com pouca ou nenhuma familiaridade com termos técnicos (mesmos os mais simples, por exemplo, exame pré-natal) e conhecimento insuficiente sobre a deficiência do filho. A análise da entrevista com o conselheiro indica um sentimento de angústia e frustração. Este se relaciona diretamente ao dever seguir a regra de não-diretividade resguardada no processo de AG:

[...] esse caso me gera muita angústia, pois você não pode dizer para a família o que fazer. Você precisa assumir uma postura não diretiva, e essa postura me deixa frustrado porque, às vezes, agiríamos de uma forma, mas não podemos interferir.

Outro fator que desperta a angústia dentro do processo de AG está relacionado à complexidade e dificuldade do AG em síndromes de má formação congênita que pode ser inferido com casos não tranquilos. Quando o aconselhamento é de consanguinidade aí eu gosto de fazer, porque são casos mais tranquilos. Agora, casos de pacientes que já chegam com a síndrome aparente, aí acho muito angustiante em serem feitos.

No que ser refere ao sentimento do conseIheiro durante o processo de AG, a análise aponta, novamente, a presença da angústia decorrente da disposição afetiva do conseIheiro se identificando com o sofrimento da consulente. Bem, como eu já Ihe havia falado, com muita angústia. Eu tento tranquilizar a mãe, porque, essa sim, eu percebo que mais sofre com o diagnóstico e é ela que, na maioria das vezes, vem à procura do $A G$.

Pode-se notar, ainda, uma tentativa de preparação do conselheiro para lidar com a situação do AG. Neste sentido, o conselheiro informa que, antecedendo ao processo, ele realiza uma ampla pesquisa sobre o diagnóstico e a síndrome e procura expor alternativas de tratamento, lugares de apoio e indicações médicas, focando nos aspectos positivos do desdobramento da síndrome visando tranquilizar a consulente.
Outro elemento importante se refere à percepção, por parte do conselheiro, de que a família necessita de tempo para a compreensão do diagnóstico: Sei que muitas vezes a família nem escuta o que estamos falando, mas é importante ser falado, porque quando eles forem embora para suas casas, ela irá começar a pensar sobre o assunto.

Sobre a percepção da reação da consulente, o conselheiro pensa que a reação (emocional) da consulente foge às suas atribuições dentro do processo de AG. Somente em caso extremo (desespero, choro), essa reação é acolhida. Então, é fornecido um tempo para a recuperação da consulente antes de ser dada sequência ao diagnóstico do processo.

Sobre a questão do tempo disponível aos consulentes e aos familiares, o conselheiro diz que a quantidade excessiva de atividades impede uma maior atribuição de tempo ao processo de AG:

Eu não sou psicólogo, com isso não fico muito voltado para a reação da mãe, somente quando elas se desesperam no consultório que dou uma parada, deixo-a chorar e continuo com o diagnóstico. [...]Temos muitas atividades durante o dia... isso atrapalha um pouco o AG, pois não podemos prestar maior tempo aos pacientes.

Em relação às considerações do conselheiro sobre a atividade do AG, pode-se afirmar que este percebe a equipe ainda em uma fase de formação. Salienta a importância de um foco multidisciplinar no processo do AG e informa sobre a necessidade de um respaldo, por parte de outros profissionais (médicos, fisioterapeutas, psicólogos, terapeutas ocupacionais, biomédicos, biólogos etc.), para o atendimento em AG. No entanto, embora pense na importância da multidisciplinaridade ao discorrer sobre as funções de cada profissional, pode-se perceber que a ação deste é concebida de forma não integrada. Salienta, ainda, o excesso de atividade dos profissionais como fator que se contrapõe a prestar um melhor serviço aos pacientes. 
Quanto aos resultados da observação da seção devolutiva do AG, ao iniciar o processo, o conselheiro segue o protocolo e questiona sobre os motivos que as levaram a buscar o serviço de AG. Pode-se perceber, na resposta da consulente, uma dificuldade em compreender/gerir a necessidade especial do filho. Fala do cansaço e da frustração em relação aos cuidados básicos e referese à educação como algo sem sentido e com um caráter simplesmente obrigatório. Percebe-se, também, uma incapacidade de obter ajuda profissional específica para as dificuldades do filho, além do desconhecimento sobre a capacidade potencial do desenvolvimento delas.

A segunda seção está relacionada à gravidez da consulente. Ela conta que foi tranquila, mas que "[...] não fez esse negócio que faz na gravidez" (pré-natal). Pode-se perceber um vocabulário restrito, com pouca familiaridade com termos técnicos e a ausência de assistência no período de gestação. Pode-se, ainda, inferir que a consulente é uma pessoa com um nível de pensamento simples e carente de esclarecimentos e de assistência.

Na terceira seção, a entrevista é direcionada para os resultados do diagnóstico. O conselheiro os lê. O resultado é positivo para a síndrome de 47, XYY. O conselheiro discorre sobre as alterações decorrentes da síndrome: "alteração de comportamento, dificuldade de aprendizagem, dificuldade na fala, entre outras alterações".

Exposta ao diagnóstico, a consulente passa a evitar contato visual, morde os lábios, entrelaça as mãos expressando surpresa e sinais de tensão emocional. Após alguns minutos, a consulente começa a falar sobre os prejuízos causados pelo filho, sobre os conflitos gerados com o marido em função desta situação, da culpa que lhe é imposta, da percepção do marido e das pessoas de que ela não cuida do filho. Refere-se ao cuidar como obrigação imposta pelo conselho tutelar e que a educação não vale a pena fornecendo indicações de uma inabilidade para lidar com a crise vital e o uso de meios agressivos em sua criação.
Destaca-se uma percepção sobre o filho, a qual a mãe o considera como alguém que não sabe o que faz, não pensa nada e que apresenta uma agressividade excessiva na conduta. Portando, a consulente questiona o conselheiro sobre a capacidade de desenvolvimento do filho. Em resposta recebe a seguinte informação: "ele não vai ter uma vida normal como as outras crianças, mas ele precisa ser estimulado, que quem sabe um dia ele pode trabalhar como garçom".

Na quarta seção, seguindo o protocolo, o conselheiro questiona: "o médico de $\mathrm{N}$. explicou para a senhora o que ele tem?" A consulente responde que, "[...] segundo o médico, o que aconteceu foi na hora dele nascer, que aconteceu alguma coisa que deu errado". O fragmento de fala indica conhecimento limitado da mãe para que possa elaborar uma resposta sobre as condições que poderiam ter favorecido a causa da deficiência do filho, além de um relato que traz uma concepção incorreta e pouco esclarecedora do que pode ter sido informado pelo seu médico assistente.

Prosseguindo, o conselheiro questiona a presença na família de outros indivíduos com problemas genéticos. A resposta revela um total desconhecimento sobre a condição do filho e a incompreensão do termo técnico "genético": "se é genético, minha família nunca comentou!" Ao término do processo de aconselhamento, o conselheiro solicita que a consulente retorne ao médico assistente do filho para que este efetue a troca do medicamento e o encaminhe para estimulação. A consulente se levanta e deixa a sala em silêncio.

\section{Discussão}

Realizou-se a discussão sobre a sessão de AG a partir do cotejamento a quatro temas centrais do aconselhamento psicológico: o acolhimento, a comunicação, a não-diretividade e a crise vital. Pode-se perceber que o tópico acolhimento não é realizado em nenhum momento da sessão. O conselheiro se orienta somente pelo protocolo do AG e deixa passar várias oportunidades de intervir 
no sentido de acatar a incerteza, a angústia, o desamparo, a culpa e o conflito da consulente frente ao diagnóstico, e demonstra inabilidade, conduta inadequada (agressiva) e omite informações à consulente sobre como agir com o filho. Neste sentido, perde-se um dos focos centrais do processo de AconseIhamento como possibilidade de acolher o campo existencial do sujeito.

Conforme o tema comunicação, pode-se verificar que, submisso ao protocolo, o conselheiro faz uso de linguagem técnica não acessível à compreensão da consulente. Perde-se, assim, em vários momentos, a oportunidade de abordar temas que referese à relação dos familiares com o problema diagnosticado. A comunicação não integrada à fala da consulente não estabelece contato com os temas e questões centrais que se presentificaram no desenrolar do processo de aconselhamento. Resumindo: um colóquio sem interlocução.

Como pode ser compreendido, a não-diretividade se constitui como princípio ético central do aconselhamento genético. Percebe-se a ação do conselheiro de se submeter ao protocolo como uma forma de resguardar esse princípio. Contudo, da forma que foi colocada em prática, a não-diretividade termina por restringir a comunicação do conselheiro, comprometendo o esclarecimento de informações e das questões inquiridas pela consulente.

Apenas em um momento, após o relato do fracasso escolar, o conselheiro responde às incertezas da consulente e nesse momento ele é incorretamente diretivo ao restringir a potencialidade da criança a uma atividade específica. Por conseguinte, é o momento crucial do destino da consulente e seus familiares. Assim, a crise vital que se instala no caso de um diagnóstico positivo se constitui como elemento fundamental a ser gerido pelo processo de Aconselhamento. Desse modo, ao ser realizado o diagnóstico, observam-se sinais de incerteza, desinteresse afetivo, tensão emocional, angústia e negação; sinais expressivos da crise vital que se instala.
No presente estudo, observa-se que, por comparação dos registros da sessão de AG com os registros da entrevista do conselheiro e da consulente, a angústia inerente às crises vitais pode ser vivenciada nos dois polos (profissional/consulente-familiar) da relação de aconselhamento. Deste modo, pode-se inferir que os aspectos contraproducentes observados nessa experiência de AG podem resultar em um processo de identificação gerador de angústia e de defesas como forma de reatividade psíquica.

Considerando-se a entrevista com o conseIheiro, pode-se ressaltar que este se encontra alinhado sob uma perspectiva teórica com os princípios éticos e técnicos do AG (Silva, \& Ramalho, 1997; Pina-Neto, 2008). Conclui-se que, além de um preparo intelectual e técnico, torna-se necessário a busca por uma preparação afetiva para a função de conselheiro.

\section{Considerações finais}

O aconselhamento é, portanto, definido como um processo de investigação clínica de uma condição genética e que visa à orientação sobre o diagnóstico e riscos de ocorrências/ recorrências para as famílias e/ou para os consulentes. Esta definição se centra na informação genética. Contudo, a partir da observação do processo de AG, pôde-se verificar que este extrapola os limites desta tarefa, envolvendo uma relação intersubjetiva na qual as informações de ordem genética não são emocionalmente neutras, podendo suscitar angústia, temores, culpas e outros sentimentos que influenciam, profundamente, as decisões pessoais dos consulentes e dos seus familiares.

O AG se constitui como um processo de extrema complexidade, impactando o ciclo existencial do consulente e de sua família. Nesse sentido, a prática pelo profissional do AG deve resguardar-se por uma conduta que envolve não apenas aspectos de preparação teórica e técnica, mas, também, a capacidade de lidar com aspectos afetivos advindos do desempenho de suas funções. É importante que os profissionais do AG tenham habilidades para que possam compreender os consulentes 
e lhes forneçam o tempo necessário para assimilarem as informações e tomarem decisões necessárias com maior clareza e racionalidade. É preciso que o profissional compreenda a dor dos consulentes e respeite o momento de cada família.

O acolhimento, por parte dos conselheiros, é facilitador do esclarecimento dos fatores biológicos, comportamentais e emocionais que proveem das crises vitais. Essa atitude de acolhimento pressupõe que se disponha a esclarecer ao consulente e aos familiares, não apenas o diagnóstico, mas, também, a melhor forma para lidar com ele.

Acolher, contudo, não consiste em somente aliviar o sofrimento do consulente, mas ajudá-los a se expressarem sobre os aspectos dos seus problemas e direcionar este sofrimento para canais construtivos. A comunicação deve ser um processo de duas vias. O conselheiro não deve apenas fornecer informações, mas principalmente estar receptivo às expressões dos elementos cognitivos e emocionais do consulente e/ou familiares; e deve estabelecer uma linguagem acessível e empática centrando-se no nível de compreensão que o consulente e os familiares possuem.

Não menos importante, o princípio ético do aconselhamento preconiza ao conselheiro uma postura não-diretiva distinta daquela adotada na relação médico-paciente. A mudança de posição de sujeito-do-saber para facilitador da informação genética exige uma sensibilidade permanente do conselheiro frente às suas opiniões, crenças e juízos de valores.

Os dados da pesquisa apontam a importância de se considerar os fatores emocionais que se seguem a um diagnóstico positivo. Pôde-se perceber, na fala da consulente, sinais de angústia, temores, culpas, incertezas, desconhecimento e incapacidade operacional de gerenciamento da crise vital específica; mais ainda, o distanciamento afetivo e o lugar da criança no discurso da mãe como criança-problema.

Compreende-se que o momento do AG deveria constituir-se como o espaço para o acolhimento de todas essas questões. Percebe-se, portanto, que, no caso específico da pesquisa, o processo não conseguiu estabelecer-se como possibilidade de escuta para essas demandas.

É importante destacar que o AG é uma especialidade em desenvolvimento, e que, na atualidade, se encontra sob as diretrizes do campo das ciências biológicas. São insuficientes os trabalhos que incluam, nesse contexto, o entendimento da dinâmica psicológica envolvida, bem como das técnicas e manejo, visando alcançar os objetivos do processo de aconselhamento sob aspectos da informação, da continência e do esclarecimento.

Na teoria e na prática, a Psicologia possui uma vasta experiência relacionada ao processo de aconselhamento em diversificadas situações. Supõe-se que a inserção do conhecimento psicológico possibilitaria ao AG:

1. Trabalhar em conjunto com a equipe multiprofissional visando:

- Fomentar uma preparação ao nível da dinâmica psicológica da própria equipe envolvida no processo de AG; e

- Fomentar uma preparação no nível da dinâmica psicológica envolvidas nas crises vitais advindas do processo de AG, buscando desenvolver a percepção dos fenômenos emocionais, seja para um melhor acolhimento do consulente, seja para o encaminhamento nos casos em que se julgar necessário um apoio psicológico.

2. Trabalhar junto com a equipe multiprofissional no processo de AG visando:

- O acolhimento do sofrimento e da demanda do consulente e familiares;

- A identificação das preocupações fundamentais que os consulentes e familiares apresentam; e

- A promoção de comportamentos e enfrentamentos das situações afins ao estabelecimento do desenvolvimento e bem-estar do consulente e dos familiares. 
Os elementos assinalados são os que apresentam importância significativa para a presente pesquisa.

Compreende-se que o mérito desta investigação reside no fato de destacar a importância de serem considerados os aspectos emocionais presentes e eliciados no processo de AG. Contudo, não se pode deixar de vislumbrar em trabalhos futuros a necessidade de uma metodologia que busque ampliar a amostra tanto no que se refere ao quantitativo de casos e número de membros do núcleo familiar como, também, a investigação do AG em outras síndromes.

Considera-se, por fim, que, quando houver a integração da Psicologia ao processo de AG, novos elementos poderão, com certeza, enriquecer tanto a ciência da Psicologia quanto a ciência do Aconselhamento Genético. Este é o campo de ação, potencialidades e desejos que surge diante dos psicólogos. 


\section{Juliana Santos Souza Hannum}

Doutoranda pela Pontifícia Universidade Católica de Goiás - GO. Brasil.

E-mail: dra.julianahannum@yahoo.com.br

\section{Fábio Jesus Miranda}

Doutor pela Pontifícia Universidade Católica de Goiás - GO. Brasil.

\section{Luciana Novais de Oliveira Brito}

Mestre pela Pontifícia Universidade Católica de Goiás - GO. Brasil.

\section{Sebastião Benício da Costa Neto}

Doutor pela Pontifícia Universidade Católica de Goiás - GO. Brasil.

\section{Aparecido Divino da Cruz}

Doutor pela Pontifícia Universidade Católica de Goiás - GO. Brasil.

\section{Endereço para envio de correspondência:}

Pontifícia Universidade Católica de Goiás (PUC-GO). Avenida T 05 Qd. 117 Lt. 07, 149. Setor Bueno. CEP: 74230-045. Goiânia - GO. Brasil.

Agradecemos ao Fundo de Amparo à Ciência e Tecnologia do Estado de Goiás (FAPEG) pela concessão de bolsa de mestrado que permitiu a realização do presente artigo.

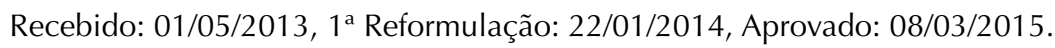


Referências
Bardin, L. (2008). Análise de conteúdo. Lisboa: Edições 70.

Bleiker, E. M. A., Hahn, D. E. E., \& Aaronson, N. K. (2003). Psychosocial issues in cancer genetics: current status and future directions. Acta Oncologia, 42(4), 276-86. doi:10.1080/02841860310004391

Borges-Osório, M. R., \& Robinson, W. M. (2001). Genética humana (2a ed.). Porto Alegre, RS: Artmed.

Brasil (1996), Ministério da Saúde, Conselho Nacional de Saúde. Resolução no 196, de 10 de outubro de 1996. [Aprova as diretrizes e normas regulamentadoras de pesquisas envolvendo seres humanos]. Brasília, DF: Ministério da Saúde, 1996.

Brunoni, D. (2002). Aconselhamento genético. Ciência \& Saúde Coletiva, 7(1), 101-107. doi:10.1590/S1413-81232002000100009

Colmago, N. A., \& Biasoli-Alves, Z. M. M. B. (2004). Orientação familiar para pais de crianças com síndrome de Down. Revista de Extensão e Pesquisa em Educação e Saúde, 2(2), 11-29

Evers-Kiebooms, H., \& Berghe, H. (1979). Impact of genetic counseling a review of published follow-up studies. Clinical Genetics, 15(6), 465-474. doi:10.1111/j.1399-0004.1979.tb00827.x

Garicochea, B., \& Barros, M. C. M. (2008). $\mathrm{O}$ aconselhamento genético em câncer. In M. J. Kovacs, M. H. P. Franco, V. A. Carvalho (Orgs.), Temas em psico-oncologia (pp. 23-31). São Paulo, SP: Summus.

Goldberg, S., Morris, P., Simmons, R., Fowler, R., \& Levison, H. (1990). Chronic illness infancy and parenting stress: a comparison of three groups of parents. Journal of Pediatric Psychology, 15(3), 347-358. doi:10.1093/jpepsy/15.3.347
Irvin, N., Kennel, J., \& Klaus, M. (1993). Atendimento aos pais de um bebê com mal formação congênita. In: Klaus, M., Kennel, J. (1993), Pais/bebê: a formação do apego (pp. 245-275). Porto Alegre, RS: Artes Médicas.

Kroeff, C., Maia, C., \& Lima, C. (2000). O luto do filho malformado. Femina, 28, 395-396.

Mc Kusick, V. (1975). Genetic counseling. American Journal of Human Genetics, 27(2), 40-42.

Pelchat, D., Ricard, N., Bouchard, J-M., Perreault, M., Saucier, J-F., Berthiaume, M. et al. (2001). Adaptation of parents in relation to their 6-month-old infant's type disability. Child: Care, Heath and Development, 25(5), 377398. doi:10.1046/j.1365-2214.1999.00107.x

Petean, E. B. L., \& Pina-Neto, J. M. (1998). Investigação em aconselhamento genético: impacto da primeira noticia: reação dos pais à deficiência. Revista Medicina (Ribeirão Preto), 31(2), 288-295.

Pina-Neto, J. M. (2008). Aconselhamento genético. Jornal de Pediatria (Rio de Janeiro),84(suppl. 0), 20-26. doi:10.1590/S0021-75572008000500004

Ramalho, A. S., Magna, L. A., \& Silva, R. B. P. A. (2002). A Portaria MS n. ${ }^{\circ} 822 / 01$ e a triagem neonatal das hemoglobinopatias. Revista Brasileira de Hematologia e Hemoterapia, 24(4), 244-250. doi:10.1590/S1516-84842002000400002

Rim, P.H.H., Magna, L.A., \& Ramalho, A. S. (2006). Genetics and prevention ofblindness. Arquivos Brasileiros de Oftalmologia, 69(4), 481-485. doi:10.1590/S0004-27492006000400005

Silva, R. B. P., \& Ramalho, A. S. (1997). Riscos e benefícios da triagem genética: o traço falciforme como modelo de estudo em uma população brasileira. Cadernos de Saúde Pública, 13(2), 285-294. doi:10.1590/S0102-311X1997000200018 